\title{
Corona Discharge Velocimeter
}

\author{
Philippe Béquin ${ }^{1)}$, Vincent Joly ${ }^{1)}$, Philippe Herzog ${ }^{2)}$ \\ 1) Laboratoire d'Acoustique de l'Université du Maine - UMR CNRS 6613 \\ Université du Maine, 72085 Le Mans Cedex 9, France. philippe.bequin@univ-lemans.fr \\ 2) LMA, CNRS, UPR 7051, Aix-Marseille Univ, Centrale Marseille, \\ 13453 Marseille Cedex 13, France. herzog@lma.cnrs-mrs.fr.
}

May 16, 2018

\section{Summary}

An acoustic velocity sensor is described. It uses a small volume of ionized gas as sensing element to estimate the velocity of air particles, especially their oscillations caused by an acoustic perturbation. The generation of charged particles is performed by a negative point-to-plane discharge, the plane anode being split into two electrically insulated parts. The principle of this velocity sensor is based on the measurement of the radial deflection of the charged particles which flow between the point and the two half-planes, this deflection being caused by the motion of the air particles resulting from the acoustic field. An electroacoustic model of this velocity sensor is proposed, based on the fluctuations of the current density distribution over the plane electrodes. An experimental set-up is developed using a waveguide. It allows to compare simultaneously the acoustic velocity deduced from the corona discharge sensor on one side, and that resulting from the two-microphone meth od on the other side. This paper also proposes a parametric study to quantify the influence of the electrical and geometrical parameters of the discharge on this acoustic sensor.

PACS no : 51.40.+p, 52.80.Hc,52.80.-s, 43.38.+n, 43.58. $+\mathrm{z}$

\section{Introduction}

In acoustics, the particle velocity can be obtained by various methods and measuring devices. The most well-known are :

The two-microphones method $[11,15,16]$ : Estimation of the acoustic particle velocity is based on the finite difference approximation of pressure gradient by using a pair of well-matched pressure microphones.

The hot wire anemometer [30] : In this device, a tiny wire attains an equilibrium temperature when heat generated in it is just balanced by convective heat loss from its surface. Air flow oscillations modify its cooling, inducing a change in the resistance of the wire.

The double hot-wire sensor developed by Microflown ${ }^{\circledR}[32,36]$ : As above, a particle velocity signal in the direction perpendicular to the wires changes their temperature. The upstream wire is cooled more than the downstream wire by the air flow. The resulting resistance difference is proportional to the particle velocity.

The Laser Doppler Anemometry [4, 12, 33, 39] : In the measurement volume delimited by the crossing of a split laser beam, air is seeded with particles capable to follow the acoustic oscillations. Light is scattered by theses particles, which are moving relatively to the light source. The Doppler effect induces a frequency shift which is demodulated by comparison with the reference laser beam.

The Particle Image Velocimetry (PIV) [21, 24] : A pulsed laser beam creates a light sheet in a measurement plane, where air is seeded as above. Correlation of pictures taken at short time intervals allows to retrieve a velocity map.

These methods and devices have advantages and drawbacks [35] which are not discussed in the present paper.

The gas-discharge velocimeter described here is an acoustic particle velocity sensor derived from the anemometer of Lindvall [1]. Later work on the ionic anemometers was carried out by several authors $[5,6,18,19,20,23,31,40,41]$ in order to design probes for specific applications. However all these ionic devices are dedicated to the measure of the fluid flow velocity : they estimate average velocity and fluctuations associated with turbulence.

The objective of the present work is to propose an ionic sensor adapted to the measurement of 
the acoustical phenomena. This study comes in complement to the study carried out by the same authors on a gas-discharge microphone [37, 38]. Note that the association of these two gas-discharge sensors would thus allow the measurement of the acoustic pressure and the acoustic particle velocity within the same small volume of ionized gas ; this is an important property desirable e.g. in the design of a sound intensity probe.

This paper begins with a description of the corona discharges velocimeter. Sections 3 and 4 propose specific models that describe respectively the electrical and acoustical behaviors of this velocimeter. Section 5 describes the experimental setup and the measurements performed ; in section 6.1 the results from the corona discharge velocimeter (CDV) are compared in a waveguide with those obtained from the twomicrophones method. The remainder of the paper (Sec. 6.2) is devoted to a parametric study ; influence of the electrical and geometrical parameters of the discharge on the sensitivity of the corona discharges velocimeter is analyzed. Concluding remarks and further investigations are given in Section 7 .

\section{Principle and design of the corona discharge velocimeter}

The principle and design of the corona discharge velocimeter (CDV) are illustrated by figure 1. It is based on the detection of the movements of ions affected by the displacement of the air particles due to a sound wave [37]. The generation of these ions is performed by a negative point-to-plane corona discharge.

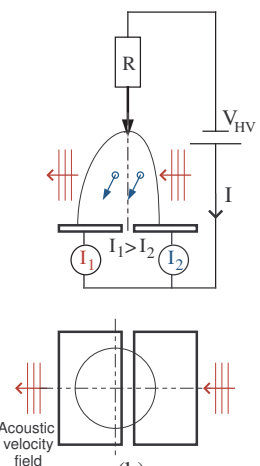

(b)
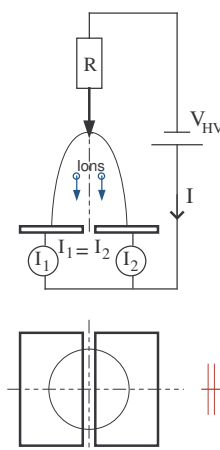

(a)
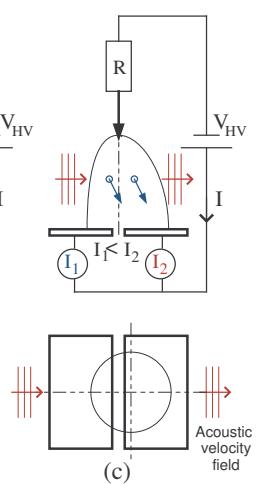

Figure 1: Schematic representation of a point-toplane corona discharge velocimeter. (a) without acoustic perturbation ; $(\mathrm{b}, \mathrm{c})$ with an acoustic velocity field.

The planar anode is split into two symmetrical parts. They are electrically insulated one from the other by a small gap ( $\simeq 1 \mathrm{~mm})$. Without acoustic disturbance (figure 1a), the corona discharge is axially symmetrical and equal currents flow through the two anodes $\left(I_{1}=I_{2}\right)$. When the ionized gas is traversed by an acoustic disturbance with a direction of propagation of the sound differing from the trajectory of the ions (figure 1b, 1c), their velocity has a transverse component (horizontal in figure 1). Each anode part then receives alternately more or less electric charges than the other $\left(I_{1} \neq I_{2}\right)$. The difference between the currents collected by the anodes therefore reflects the fluctuations in particle velocity associated with the transverse component of the acoustic perturbation.

\section{Negative point-to-plane corona discharge}

When a high voltage is applied between a point and a plane (figure 2), charged particles are created and interact with neutral particles of the gas. The flow of these particles gives rise to an electric current in the external electric circuit. For a given range of voltages $V_{H V}$ above a threshold $V_{s}$, the electric current $I$ consists in regular pulses (known as "Trichel pulses"), with a repetition rate around a few hundred kilohertz (far higher than audio frequencies: $20 \mathrm{~Hz}-20 \mathrm{kHz}$ ). As the voltage is increased, the pulses turn into a steady-state current regime (glow discharge) until a spark occurs $[2,8,10,14,13,26]$. In the "Trichel pulses" regime, the frequency of the pulses depends on various factors such as the geometrical and electrical configurations of the electrodes but also on the surface properties.

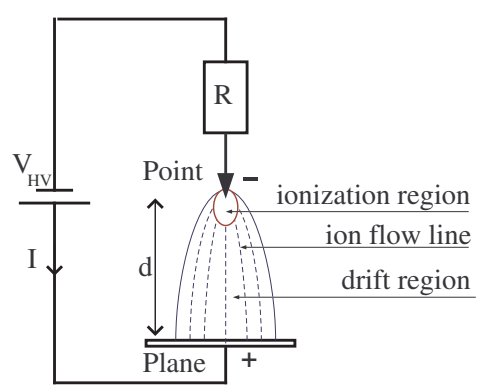

Figure 2: Schematic representation of a point-toplane discharge.

For this regime, a simple quadratic relation allows to model the dependence of current $I$ with the voltage $V_{H V}[10,17,26,27]$ :

$$
I=C \mu_{i} V_{H V}\left(V_{H V}-V_{s}\right) \quad[A],
$$

where $C$ is a factor which depends on the geometry and the material of the electrodes, $\mu_{i} \simeq 1.810^{-4} V^{-1} m^{2} s^{-1}$ is the negative ion mobility in air, and $V_{s}$ is the threshold voltage 
Table 1: Parameters associated with the experimental data (characteristics $I-V_{H V}$ of Figure 3). The values of the parameters $V_{s}$ and $C \mu_{i}$ with their uncertainties are provided by $I / V_{H V}=C \mu_{i}\left(V_{H V}-V_{s}\right)$ using a minimization routine.

\begin{tabular}{llllll}
\hline$d$ & {$[m m]$} & 5 & 7 & 9 & \pm 0.3 \\
\hline$V_{s}$ & {$[k V]$} & 2.7 & 2.9 & 3 & \pm 0.3 \\
$C \mu_{i}$ & {$\left[p A V^{-2}\right]$} & 3.2 & 1.9 & 1.1 & \pm 0.1 \\
\hline
\end{tabular}

associated with the discharge start-up. Note that the actual corona discharge is a fast timedependent phenomenon, involving timescales much shorter than the acoustic phenomena considered here. The classical simplified model presented here (Eq. 1) therefore describes the discharge as a stationary process, involving quantities which are time averaged over the discharge pulses period to keep only fluctuations related to the acoustic velocity.

Figure 3 gives a representative example of currentvoltage characteristics deduced from measurements carried out on point-to-plane configurations close to those used in the corona discharge velocimeter. Using the linear relation $I / V_{H V}=C \mu_{i}\left(V_{H V}-V_{s}\right)$, the threshold voltage $V_{s}$ and the quantity $C \mu_{i}$ (see table 1 ), useful for the calculation of the sensitivity of the velocimeter, are deduced from the parameters of the least-squares fitted lines.

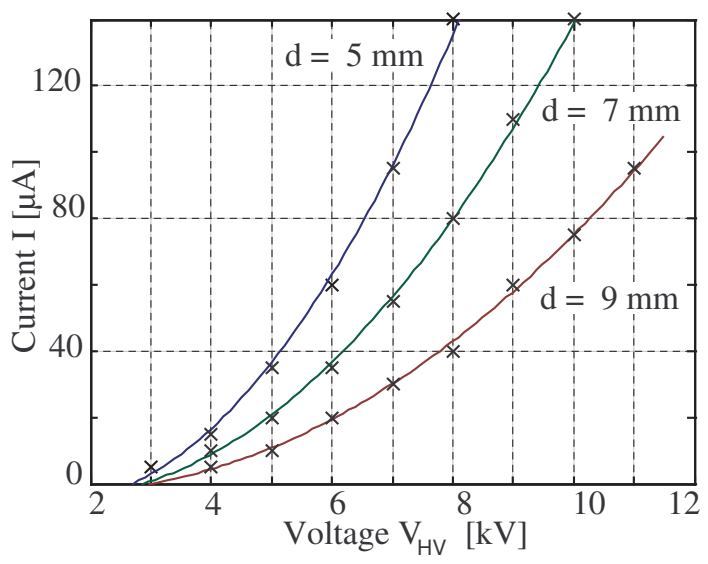

Figure 3: Variation of current $I$ with voltage $V_{H V}$ associated with a point-to-plane discharge with a gap length $d$ (figure 2). Comparison between experimental data $(\times \times \times)$ and analytical model - Equation 1 - (-) with the adjusted parameters $V_{s}$ and $C \mu_{i}$ deduced from a least-squares method (Table 1).

\section{Physical model}

The gap between the point and the plane can be divided into two parts (figure 2) : (i) a charged particle production region in the vicinity of the cathode where the electric field is high and in which ionization processes dominate; (ii) a drift region which occupies most of the gap in which the electrons from the ionization region move to the anode. The most energetic electrons directly reach the plane, while less energetic ones combine with neutral particles to form relatively heavy negative ions.

These heavy ionised particles drift under the action of the electric field and exchange momentum with the neutral particles during collisions: the momentum of the charged particles is thus acquired from the electric field and then transferred to the neutral particle through collisions. The associated macroscopic effect is a steady flow that blows from the point to the plane anode [3, 25, 28, 29, 42]. At the same time and in directions perpendicular to the point-to-plane axis, the momentum of the neutral particles is drawn from the acoustic field and is transferred to the cloud of charged particles by means of collisions. This leads to a deflection of the ionised cloud in a direction perpendicular to the point axis. Because the electron masses are much smaller than those of the neutral particles, their macroscopic motion is marginally affected by the movement of the neutral particles ; consequently, the information on the acoustic velocity of the perturbation is considered to be only carried by the negative ions.

The development of a simple model which expresses current variation $i(t)$ in terms of acoustic velocity $v_{a}(t)$ requires several assumptions :

- The acoustic velocity field is considered as uniform within the whole volume of ionized gas ; consequently the dimensions of the point-toplane discharge must be small compared to the considered acoustic wavelengths.

- In the drift region (the sensitive region), the velocity of the negative ions $\vec{V}$ is assumed to be the vector superposition of their drift velocity $\overrightarrow{V_{i}}=\mu_{i} \vec{E}(\vec{E}$ is the electric field inside the drift region) and the sensed acoustic velocity component $\overrightarrow{v_{a}}$ which is directed perpendicular to the axis of the discharge (see figure 4).

- The behavior and the path of the electrons are not significantly modified by the movement of the neutral particles.

- The time of flight time of the negative ions through the sensitive region must be shorter than the timescale associated with $\overrightarrow{v_{a}}$. Equivalently, 


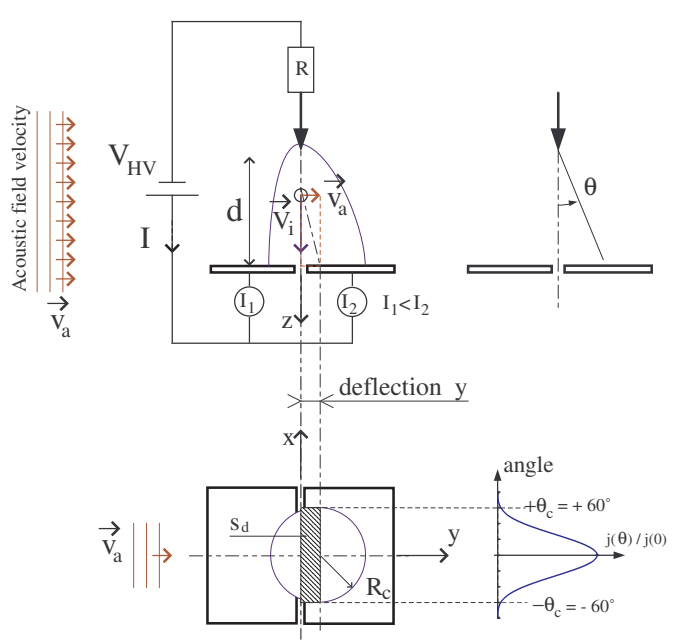

Figure 4: Schematic representation of negative ions deflection $y$ associated with the acoustic perturbation. $\frac{j(\theta)}{j(0)}$ is the relative current density on the plane electrodes. $R_{c}=d \tan \left(\theta_{c}\right)$ is the radius of the circular area impinged by the charged particles.

the relation $\left|\overrightarrow{v_{a}}\right|<<\left|\vec{V}_{i}\right|=\mu_{i}|\vec{E}|$ gives an upper limit to the frequency range of the corona discharge velocimeter.

- The local electric field $\vec{E}$ is marginally modified by the distribution and motion of the charged particles.

\subsection{Deflection of the negative ions}

We first consider a cloud of negative ions drifting with constant mobility $\mu_{i}$ in a time-independent uniform electric field $\vec{E}$ (figure 4 ). For a given corona voltage $V_{H V}$ the velocity along a field line is roughly approximated by $V_{i}=\mu_{i} E \simeq \mu_{i} V_{H V} / d$ with $d$ the gap length. The cloud of negative ions is exposed to an acoustic perturbation with an acoustic velocity component $\overrightarrow{v_{a}}$ perpendicular to $\vec{V}_{i}$ and it undergoes a deflection $y$ on the plane electrodes. Assuming a streamline fluid flow allows to deduce the deflection :

$$
y=\frac{v_{a}}{V_{i}} L \quad[m],
$$

where $L$ is the distance covered by the negative ions. Note that the actual electric field $\vec{E}$ is probably not uniform over the gap; equation 2 is thus a first-order approximation which will be adjusted later.

\subsection{Current variation on an half- electrode}

The consequence of the deflection of negative ions due to acoustic disturbance is the arrival of a larger number of ions on one half anode to the detriment of the other one, causing an electric current difference between the two electrodes. The current which flows between the point and the plane electrodes is the sum of the current $I_{i}$ carried by the negative ions (sensitive to the acoustic field) and of the current $I_{e^{-}}$carried by electrons (which path is assumed unchanged) :

$$
I=I_{i}+I_{e^{-}}=I_{i}(1+\beta) \quad \text { with } \quad \beta=\frac{I_{e^{-}}}{I_{i}} .
$$

Taking into account this assumption, the variation of current $i_{v}$ measured on a plane half-electrode is only related to the current carried by the negative ions :

$$
I_{i}=\frac{I}{1+\beta} \quad[A]
$$

The total current collected by the two electrodes is classically described as $[10,14,17]$ :

$$
I=\iint_{S} j(\theta) d S=\iint_{S} j(0) \cos ^{5}(\theta) d S,
$$

where $j(\theta)$ and $j(0)$ are the current densities on the plane electrodes and at the center of the anodes respectively (figure 4), with $\tan (\theta)=r / d$ where $r$ is the spread radius at the plane electrode and $d$ is the gap length.

The negative ions which pass through an electrode instead of the other distribute over a more or less important surface according to acoustic velocity (hatched surface $S_{d}$ on figure 4 ). The deflection of the cloud is sufficiently small to consider the current density roughly constant in $y$ direction ; the integration of the current density on this surface $S_{d}$ gives the variation of current $i_{v}$ :

$$
i_{v}=\frac{1}{1+\beta}\left(\int_{0}^{y} d y\right) \cdot\left(2 \int_{0}^{R_{c}} j(0) \cos ^{5}(\theta) d x\right)
$$

with $R_{c}=d \tan \left(\theta_{c}\right)$ the radius of the circular area impinged by the charged particles.

When the corona regime associated with low current is considered, the current density $j(\theta)$ falls abruptly to zero for $\theta \geq \theta_{c} \simeq 60^{\circ}$; calculating integrals in the previous expressions (Eqs 4 and 5), the current variation $i_{v}$ caused by the deflection $y$ of the ions trajectory takes the form (see appendix) :

$$
i_{v}=\frac{I}{1+\beta} \Psi \frac{y}{d} \quad \text { with } \quad \Psi=\frac{9 \sqrt{3}}{7 \pi} \simeq 0.71 .
$$

\subsection{Electroacoustic model of the corona discharge velocimeter}

Due to the acoustic perturbation in the air surrounding an electric discharge, the ionized gas is subjected 
to an instantaneous acoustic velocity $v_{a}(t)$ which leads to a variation of instantaneous current $i_{v}(t)$ observed on each half-electrode. From the equation (6), using expression of the deflection (Eq. 2) and the currentvoltage relation (Eq. 1), the sensitivity $S_{v}$ of the sensor to the acoustic velocity can be deduced :

$$
\begin{aligned}
& S_{v}=\frac{i_{v}(t)}{v_{a}(t)}=\frac{C \Psi L}{1+\beta}\left(V_{H V}-V_{s}\right) \quad[A s / m] \\
& \text { with } \quad \Psi \simeq 0.71
\end{aligned}
$$

where $V_{s}$ and $C$ are respectively the threshold voltage and a factor which depends on the geometry and the material of the electrodes (table 1), both obtained from electrical measurements. $L$ is the drift distance, $\beta=\frac{I_{e}-}{I_{i}}$ is the ratio of the currents carried by the electrons and the negative ions. The lack of information about the two multiplicative factors $\beta$ and $L$ prevents from giving explicit values for $S_{v}$. We here consider that these two factor only depend on the discharge characteristics, but not on frequency. The ratio $L /(1+\beta)$ is thus considered as a scalar factor to be determined experimentally.

As a first step, we arbitrarily chose its value assuming $\beta=0$ (no electron inside the drift region) and $\mathrm{L}=\mathrm{d}$ (the cloud of negative ions starts to derive close the point electrode) regardless of the sound wave frequency. These arbitrary assumptions lead to systematically over-estimate the actual sensitivity of the corona discharge velocimeter. A global multiplicative coefficient $K_{v}$ is then inserted, which value is adjusted to obtain the best fit with the experimental results $\left(0<K_{v} \leq 1\right)$. Replacing the unknown factor $L /(1+\beta)$ by $K_{v} d$, the expression for the sensitivity $S_{v}$ therefore takes the following form :

$$
S_{v} \simeq K_{v} \Psi C d\left(V_{H V}-V_{s}\right) \quad[A s / m] .
$$

This sensitivity is proportional to the gap length $d$, it depends on the operating point of the electric discharge $\left(I, V_{H V}\right)$ and on the parameters $C$ and $V_{s}$, quantities which are deduced from the current-voltage characteristics associated with the point-to-plane discharge.

\section{Experimental system}

\section{$5.1 \quad$ Experimental setup}

The experimental setup represented by figure 5 features three parts : (i) the high voltage supply and the corona discharge velocimeter with its electronics, (ii) the waveguide including two controlled loudspeakers, and (iii) a pair of condenser microphones used as references, followed by their conditioning amplifiers.

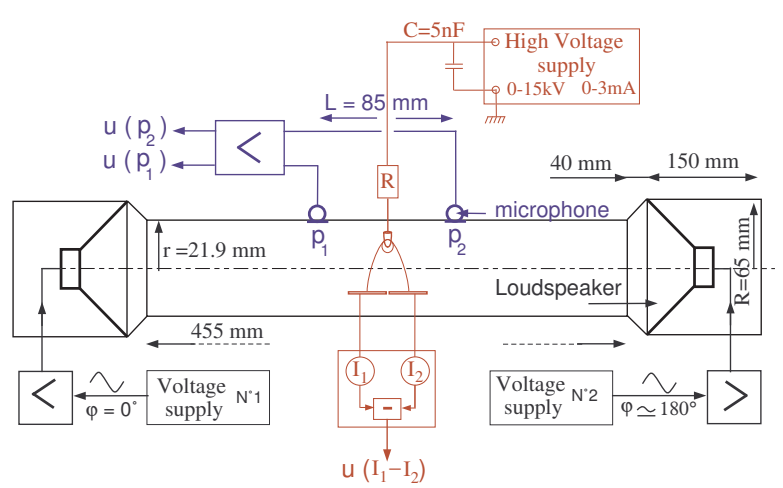

Figure 5: Experimental set-up used for the measurement of acoustic velocity.

The waveguide is a plexiglass tube with an overall length equal to $455 \mathrm{~mm}$; the two ends of the tube, via conical adapters, are connected to cylindrical PVC cavities (of radius $65 \mathrm{~mm}$ and length $150 \mathrm{~mm}$ ) containing each one a moving-coil loudspeaker (Audax HM 100 C0). Signals with opposite-phase supply the loudspeakers, so their emitted acoustic waves lead to a maximum acoustic velocity in the center of the tube and almost cancel the acoustic pressure at this position.

The propagation medium is supposed to be homogeneous and isotropic, in spite of the presence of the small volume of ionized gas due to the discharge. The diameter of the tube is supposed very small compared to the wavelength, which allows to consider a $1 D$ problem. This assumption is however limited to frequencies lower than the eigen frequency of the first higher mode [7, 22]:

$$
f \ll 1.84 \frac{c_{0}}{2 \pi r} \ll 4600 \mathrm{~Hz}
$$

where $c_{0} \simeq 345 \mathrm{~m} / \mathrm{s}$ is the speed of sound in air and $r$ is the radius of the tube.

\subsection{Estimation of acoustic velocity with a two-microphone method}

The acoustic velocity in the center of the tube is estimated with the two-microphone method [15, 16, 34]. Two condenser microphones (GRAS 40BP pressure microphone capsule + Brüel \& Kjær 2670 preamplifier, leading to a flat amplitude response up to about $70 \mathrm{kHz}$ ) are flush-mounted on the inside wall of the tube (with their protective grids removed). They are located on both sides of the axis of the corona discharge, their spacing being $L=85 \mathrm{~mm}$ (figure 5). Signals from the two microphones are fed to a conditioning amplifier (4-channel Brüel \& Kjær NEXUS) which filters them between $0.1 \mathrm{~Hz}$ and $100 \mathrm{kHz}$. Using the pressure signals $\left(p_{1}, p_{2}\right)$ delivered by these two 
microphones, the acoustic velocity on the axis of the corona discharge is deduced from the expression

$$
V_{2 m i c}=\frac{p_{1}-p_{2}}{2} \frac{1}{j \rho_{0} c_{0} \sin \left(\frac{k L}{2}\right)} \quad[\mathrm{m} / \mathrm{s}],
$$

where $\rho_{0}$ and $c_{0}$ are respectively the air density and the speed of sound ; $k=2 \pi f / c_{0}$ is the wave number. Above relation (9) only stands for $k L / 2 \neq n \pi$, which results in an upper frequency limit around $4 \mathrm{kHz}$.

\subsection{Estimation of acoustic velocity with a Corona Discharge Ve- locimeter}

The cathode system of the CDV is made up of a stainless steel needle connected in series with a resistance $R=1 \mathrm{M} \Omega$ (figure 5 ). The needle axis is placed perpendicular to an epoxy plate covered by a thin layer of tinned copper (anode) ; this layer is split into two independent parts. Since these two parts are kept at the same potential, the electric field strength close to the anode is not significantly distorted by its splitting. The values $V_{H V}$ of the voltage supply are in the range of 3 to $12 \mathrm{kV}$ with an associated DC current value $\mathrm{I}$ in the range of 0 to $0.5 \mathrm{~mA}$. All the measurements are carried out in the "Trichel Pulses" regime. A custom differential current-to-voltage amplifier followed by a low-pass filter with cut-off frequency $f_{c}=160 \mathrm{kHz}$ delivers a voltage $u$ proportional to the current difference $\left(I_{1}-I_{2}\right)$ collected by the plane electrodes.

A computer program is used to drive the frequency, amplitudes and phases of a dual-channel signal generator which supplies sinusoidal signals to the loudspeakers. A Lock-in Amplifier (SR830 DSP Stanford Research Systems) carries out a synchronous demodulation, using as its reference the generator output $n^{\circ} 1$. Its input is successively connected to each signal to measure : outputs of the microphone preamplifiers $\left[u\left(p_{1}\right), u\left(p_{2}\right)\right]$ and output of the differential amplifier conditioning the CDV $\left[u\left(I_{1}-I_{2}\right)\right]$. The measured data are then post-processed by the computer.

\section{Experimental results and dis- cussion}

Experiments reported in this section are conducted using air at atmospheric conditions : $\mathrm{P}=(1010 \pm$ 10) $10^{5} \mathrm{~Pa}, \mathrm{~T}=21 \pm 2{ }^{\circ} \mathrm{C}$ and $\mathrm{HR}=35 \pm 10 \%$. The dependance of the considered phenomena on environmental conditions has not yet been studied.

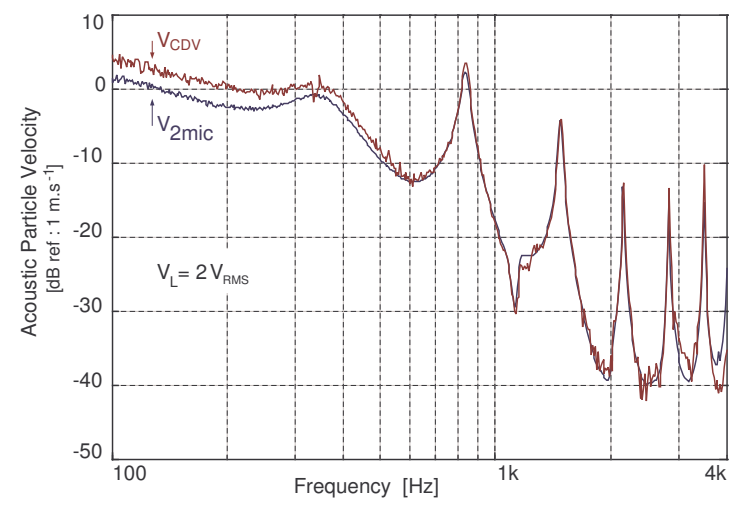

Figure 6: Acoustic particle velocity. Magnitude [dB] : $20 \log \left(v_{a} / V_{r e f}\right)$ with $V_{r e f}=1 \mathrm{~m} . \mathrm{s}^{-1} . V_{L}=2 V_{r m s}$. $V_{2 m i c}$ (blue line): two-microphone method (Equation 9). $V_{C D V}$ (red line) : corona discharge velocimeter (Equation 8) with $K_{v} \simeq 0.95, \mathrm{~d}=7 \mathrm{~mm}, V_{H V}=8$ $\mathrm{kV}, \mathrm{I}=80 \mu \mathrm{A},\left(V_{s}=2.9 \mathrm{kV}, C \mu_{i}=1.910^{-12} \mathrm{~A} \mathrm{~V}^{-2}\right)$ and $S_{v} \approx 26710^{-9} \mathrm{As} / \mathrm{m}$.

\subsection{General observations}

The figures 6 and 7 (magnitude and phase) present a representative example of the acoustic particle velocity measured in the tube with the two-microphone method (Eq.9) and the Corona Discharge Velocimeter (Eq.8). The geometrical and electrical configurations of the corona discharge are $\mathrm{d}=7 \mathrm{~mm} ; V_{H V}=8 \mathrm{kV}$, $\mathrm{I}=80 \mu \mathrm{A}\left(V_{s}=2.9 \mathrm{kV}, C \mu_{i}=1.910^{-12} \mathrm{~A} \mathrm{~V}^{-2}\right.$; $S_{v} \approx 26710^{-9} \mathrm{As} / \mathrm{m}$ ) and the voltage applied to the loudspeakers is $V_{L}=2 V_{r m s}$.

In equation $8, K_{v}$, is adjusted to best fit with results from the two-microphone method over the frequency range $[500 \mathrm{~Hz}-1 \mathrm{kHz}]$.

These figures show that the two measured velocities are similar over the whole $[100 \mathrm{~Hz}, 4 \mathrm{kHz}]$ frequency band where their comparison is valid, and the estimated levels are very close (here we used $K_{v} \simeq 0.95 \pm 0.05$, a value lower but close to unity as expected). The plot reveals the resonance of the loudspeakers loaded by their back cavity $(\simeq 340 \mathrm{~Hz})$ and anti-resonances of the tube $(\simeq 845 \mathrm{~Hz}, 1.5 \mathrm{kHz}, 2.1 \mathrm{kHz}, 2.8 \mathrm{kHz}, 3.5 \mathrm{kHz})$. In the frequency range $[100-400 \mathrm{~Hz}]$, the discharge velocimeter provides a measure of the acoustic velocity close to $1 \mathrm{~m} . \mathrm{s}^{-1}(0 \mathrm{~dB})$, a value much smaller than the upper limit $\mu_{i} V_{H V} / d \simeq 205 \mathrm{~m} \cdot \mathrm{s}^{-1}$ which is assumed for the proposed model. Figure 8 gives an another example obtained with $V_{L}=0.5 V_{r m s}$. As expected, the velocity magnitude is $12 \mathrm{~dB}$ lower than the one observed in the preceding example (figure 6 , with $V_{L}=2 V_{r m s}$ ).

The upper curve in figure 8 shows the magnitude of 


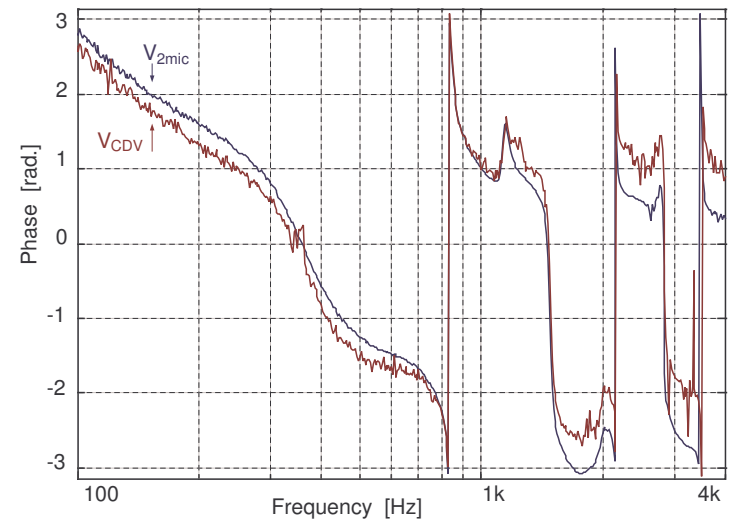

Figure 7: Acoustic particle velocity. Phase [radian]. $V_{L}=2 V_{r m s} . \quad V_{2 m i c}$ (blue line): two-microphone method (Equation 9). $V_{C D V}$ (red line) corona discharge velocimeter : (Equation 8). $\mathrm{d}=7 \mathrm{~mm}$, $V_{H V}=8 \mathrm{kV}, \mathrm{I}=80 \mu \mathrm{A},\left(V_{s}=2.9 \mathrm{kV}, C \mu_{i}=\right.$ $1.910^{-12} \mathrm{~A} \mathrm{~V}^{-2}$ ) and $S_{v} \approx 26710^{-9} \mathrm{As} / \mathrm{m}$.

the velocities ratio $V_{C D V} / V_{2 m i c}$. When the frequency decreases below about $600 \mathrm{~Hz}$ the corona discharge velocimeter response rises slightly compared to the two-microphone method ( $\simeq 3 \mathrm{~dB})$. Durbin et al. [20] also observed this discrepancy between the frequency responses of an hot wire probe and their corona anemometer (the reason for this discrepancy was however not clear). In our case, this discrepancy may be due to the acoustic displacements of air particules around the active region (production region or ionization region) which influence the equilibrium of the electric discharge and consequently the threshold voltage. As suggested by one of the reviewers, this behaviour could be empirically be taken into account by slightly varying the value of $K_{v}$ with frequency.

In addition, figures 6 and 8 also highlight that when the value of the particle velocity is lower than approximately $0.3 \mathrm{~cm} \cdot \mathrm{s}^{-1}\left(\simeq-50 \mathrm{~dB}\right.$ re m.s $\left.{ }^{-1}\right)$, the signal becomes close to the background noise of the corona discharge velocimeter. This is clearly the lower limit of the dynamic range of the mock-up probe considered in this work - although a better technology might push back this limit.

\subsection{Influence of the gap length $d$ and the applied voltage $V_{H V}$}

In order to validate the model developed previously in this paper, a parametric study is proposed : the sensitivity of the corona discharge velocimeter is studied through the values of coefficient $K_{v}$ by varying either the gap length $d$ (see Table 2) or the voltage $V_{H V}$ (see Table 3 ).

When varying the gap length $d$, the electric

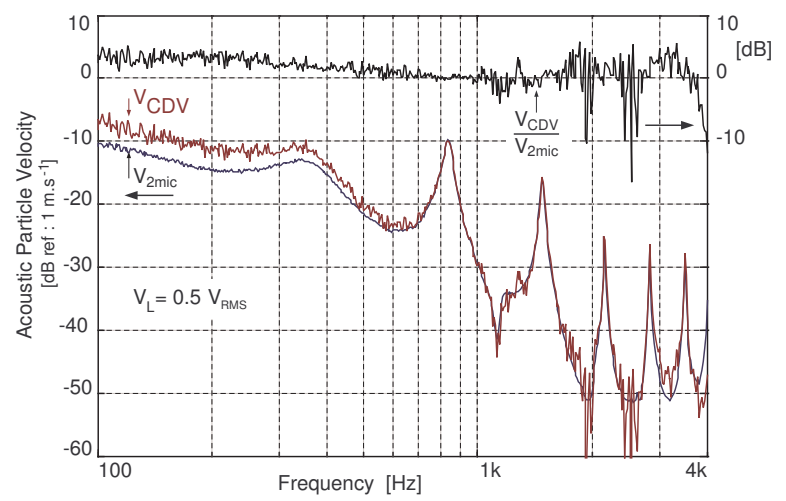

Figure 8: Acoustic particle velocity. Magnitude $[\mathrm{dB}]$ : $20 \log \left(v_{a} / V_{r e f}\right)$ with $V_{r e f}=1 \mathrm{~m} . \mathrm{s}^{-1} . V_{L}=0.5 V_{r m s}$. $V_{2 m i c}$ (blue line): two-microphone method (Eq.9). $V_{C D V}$ (red line) : corona discharge velocimeter (Eq.8) with $K_{v} \simeq 0.95, \mathrm{~d}=7 \mathrm{~mm}, V_{H V}=8 \mathrm{kV}, \mathrm{I}=$ $80 \mu \mathrm{A},\left(V_{s}=2.9 \mathrm{kV}, C \mu_{i}=1.910^{-12} \mathrm{~A} \mathrm{~V}^{-2}\right)$ and $S_{v} \approx 26710^{-9} \mathrm{~A} \mathrm{~s} \mathrm{~m}^{-1}$. Upper curve : velocity ratio $20 \log \left(V_{C D V} / V_{2 m i c}\right)$.

Table 2: Estimated value of $K_{v}$ for best fit of the Corona Discharge Velocimeter results with data from the two-microphone method. $V_{s}$ and $\left(C \mu_{i}\right)$ are deduced from the $I-V_{H V}$ characteristic of the corona discharge. $V_{L}=1 V_{r m s}$ is the voltage applied to the loudspeakers and $S_{v}$ is estimated by equation 8. The measured and predicted values are given with their estimated uncertainties (absolute and relative).

\begin{tabular}{llllll}
\hline $\mathrm{d}$ & {$[\mathrm{mm}]$} & 5 & 7 & 9 & \pm 0.3 \\
\hline$V_{H V}$ & {$[\mathrm{kV}]$} & 6.5 & 7.5 & 9.7 & \pm 0.1 \\
$\mathrm{I}$ & {$[\mu \mathrm{A}]$} & 70 & 70 & 70 & \pm 2 \\
\hline$V_{s}$ & {$[\mathrm{kV}]$} & 2.7 & 2.9 & 3 & \pm 0.3 \\
$C \mu_{i}$ & {$\left[\mathrm{pA} \mathrm{V}^{2}\right]$} & 3.2 & 1.9 & 1.1 & \pm 0.15 \\
\hline$S_{v}$ & {$\left[\mathrm{~A} \mathrm{~s} \mathrm{~m}^{-1}\right]$} & 239 & 241 & 261 & $\pm 14 \%$ \\
$K_{v}$ & {$[]$.} & 0.75 & 0.9 & 0.75 & \pm 0.05 \\
\hline
\end{tabular}

current flowing through the discharge is maintained to the value $\mathrm{I} \simeq 70 \mu \mathrm{A}$; the voltage applied to the loudspeakers is $V_{L}=1 V_{r m s}$. The table 2 gathers, on the one hand, the operating point of the discharge (voltage $V_{H V}$ and current $I$ with $V_{s}$ and $C \mu_{i}$ ) and on the other hand, the calculated sensitivity $S_{v}$ (Eq.8) and the adjusted coefficient $K_{v}$.

The coefficient $K_{v}$ is then observed to be approximately constant over the considered parameters range, with a value close to $0.8( \pm 0.05)$. The initial expression of the sensitivity $S_{v}$ (Eq. 8) with two fixed parameters $(\beta=0$ and $L=d)$ was assumed to over-estimate the sensitivity of the corona discharge velocimeter. Experimental values of $K_{v}$, lower than unity, confirm this point and provide an approximate value of the actual quantity $L /(1+\beta)$. 
Table 3: Estimated value of $K_{v}$ for best fit the Corona Discharge Velocimeter results with data from the twomicrophone method. The gap length of the CDV is $\mathrm{d}$ $=7 \mathrm{~mm} ; V_{s}$ and $\left(C \mu_{i}\right)$ are deduced from the $I-V_{H V}$ characteristic of the corona discharge. $V_{L}=1 V_{r m s}$ is the voltage applied to the loudspeakers and $S_{v}$ is estimated by equation 8 . The measured and predicted values are given with their estimated uncertainties (absolute and relative).

\begin{tabular}{llllll}
\hline$V_{H V}$ & {$[\mathrm{kV}]$} & 7 & 9 & 11 & \pm 0.1 \\
$I$ & {$[\mu \mathrm{A}]$} & 45 & 85 & 135 & \pm 2 \\
\hline$V_{s}$ & {$[\mathrm{kV}]$} & 3.7 & 4.1 & 4 & \pm 0.3 \\
$C \mu_{i}$ & {$\left[\mathrm{pA} \mathrm{V}^{-2}\right]$} & 2 & 1.9 & 1.7 & \pm 0.15 \\
\hline$S_{v}$ & {$\left[\mathrm{nA} \mathrm{s} \mathrm{m}^{-1}\right]$} & 182 & 257 & 328 & $\pm 14 \%$ \\
$K_{v}$ & {$[]$.} & 0.8 & 0.85 & 0.9 & \pm 0.05 \\
\hline
\end{tabular}

When varying $V_{H V}$, the velocimeter features a constant gap length $\mathrm{d}=7 \mathrm{~mm}$ and the voltage applied to the loudspeakers is $V_{L}=1 V_{r m s}$. For various values of the applied voltage $V_{H V}$, table 3 gathers the electrical quantities with the calculated sensitivity $S_{v}$ (Eq.8) and the adjusted coefficient $K_{v}$. As observed in the preceding part, the approximate value of coefficient $K_{v}$ is again almost constant, close to $0.85( \pm 0.05)$.

These first experimental results demonstrate that the theoretical sensitivity $S_{v}$ (Eq. 8) gives a reasonable description of the electroacoustic behavior of the Corona Discharge Velocimeter considered in this paper. The sensitivity values, depending on the geometrical and electrical configurations, are close to $25010^{-9} \mathrm{Asm}^{-1}$; in comparison, the corona anemometer developed by Durbin et al. [20] (a three needles corona discharge) presented a much lower sensitivity, close to $3810^{-9} \mathrm{As} \mathrm{m}^{-1}$.

\section{Conclusion}

This paper describes an acoustic particle velocity sensor using an weakly ionized gas ("cold plasma") as its sensing element. This ionized gas is obtained with a negative point-to-plane corona discharge in ambient air. An acoustic perturbation of the surrounding air produces a modification of the electro mechanical behavior of the plasma. A specific electroacoustic model gives an expression for the sensitivity of the sensor to the acoustic velocity, taking into account the electrical and geometrical parameters of the discharge. The lack of information on two quantities of the model $(\beta$ and L) imposes to fix their value (0 and 1 respectively) and to add to the model a factor $K_{v}$ independent of the frequency of the sound wave, allowing to adjust slightly the predictions on the experimental data.
Although the value of $K_{v}$ does not seem to vary much, this adjustment factor represents a limitation of the proposed model because its accurate determination requires a calibration of the sensor using a reference measurement system.

The implementation of the two-microphone method allowed a first comparison with measurements from this corona discharge velocimeter. The results obtained from a parametric study show globally a good agreement between the two measurement systems in the $[100 \mathrm{~Hz}-4 \mathrm{kHz}]$ frequency band. However, the comparisons between the model predictions (with the fitting factor $K_{v} \simeq 0.85( \pm 0.05)$ ) and the experimental data show that there is a difference close to $3 \mathrm{~dB}$ at lower frequencies. The model describing the interactions between the sound wave and the electrical discharges is clearly not yet complete and should be improved for better accuracy.

Nevertheless, the present study is a significant step toward the realization of an acoustic gas discharge velocimeter, and it even allows to expect the development of an impedance/intensity probe using an ionized gas for simultaneous measurement of the acoustic velocity and pressure [38].

The experimental study must be completed by varying the geometric parameters of the electric discharge (gap length $\mathrm{d}$, radius of the tip) and the material of the electrodes. Furthermore, the experimental system needs to be improved to test the sensor over a wider frequency range (reduction of the waveguide diameter). Moreover, such sensor might be miniaturized using micro discharges (micro plasma) instead of millimeter discharges $(5 \mathrm{~mm} \leq \mathrm{d} \leq 9 \mathrm{~mm})$.

\section{Appendix}

The empirical law of Warburg describing the current distribution over the plane in point-to-plane discharge can written under a reduced form [43]

$$
j(\theta)=j(0) \cos ^{5}(\theta) \quad\left[\mathrm{A} \cdot \mathrm{m}^{-2}\right]
$$

here $j(0)$ is the axial current density, $\theta=\arctan (r / d)$ the semi-vertical cone angle of the discharge as shown in figure $9, r$ the radial planar coordinate and $d$ the gap length.

The observed distributions $j(\theta)$ generally fall suddenly to zero around $\theta_{c} \simeq 60^{\circ}$ [14]. There is no clear explanation for this phenomenon other than the field lines beginning on the point and not crossing the ionization region terminate on the plane electrode outside of the radius $R_{c}$ (figure 9 ). 


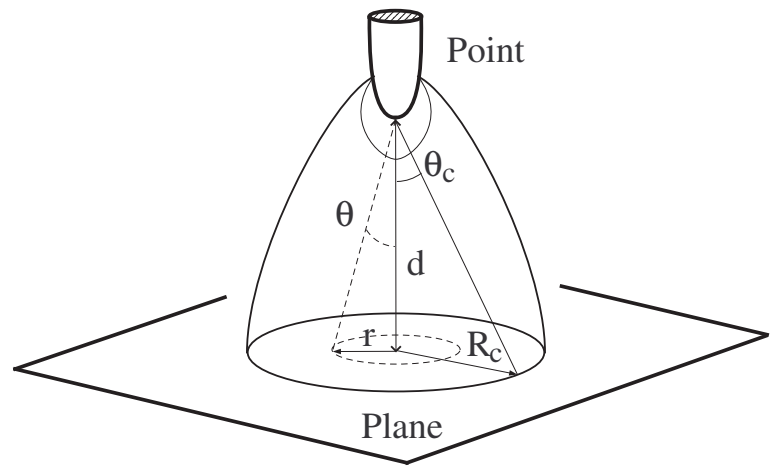

Figure 9: Schematic representation of a point-toplane corona discharge.

The integration of the current density (Eq. 10) on the circular surface of radius $R_{c}$, associated with the angle $\theta_{c}=\arctan \left(R_{c} / d\right)$, leads to the expression of the electric current :

$$
I=\iint_{S} j(0) \cos ^{5}(\theta) d S=j(0)\left(\pi R_{e f f}^{2}\right),
$$

where the radius of the effective area

$$
\begin{aligned}
R_{e f f} & =\left[\frac{2}{3}\left(1-\cos ^{3}\left(\theta_{c}\right)\right)\right]^{\frac{1}{2}} d \\
& =\sqrt{\frac{7}{12}} d \text { with } \theta_{c}=60^{\circ} .
\end{aligned}
$$

The acoustic wave causes a deflection of the ion cloud as shown in figure 4 and consequently a variation of current between the two plane electrodes (Eq. 5)

$$
i_{v}=j(0) \frac{2}{1+\beta}\left(\int_{0}^{y} d y\right) \cdot\left(\int_{0}^{R_{c}} \cos ^{5}(\theta) d x\right)
$$

with $[9]$

$$
\begin{aligned}
\int_{0}^{R_{c}} \cos ^{5}(\theta) d x & =d \int_{0}^{\theta_{c}} \cos ^{3}(\theta) d \theta \\
& =d\left[\sin \left(\theta_{c}\right)-\frac{1}{3} \sin ^{3}\left(\theta_{c}\right)\right] .
\end{aligned}
$$

Finally, the current variation can be written [37]

$$
\begin{aligned}
i_{v} & =j(0) \frac{2 y d}{1+\beta}\left[\sin \left(\theta_{c}\right)-\frac{1}{3} \sin ^{3}\left(\theta_{c}\right)\right] \\
& =\frac{I}{1+\beta} \Psi \frac{y}{d}
\end{aligned}
$$

where

$$
\begin{aligned}
\Psi & =\frac{3}{\pi} \frac{\sin \left(\theta_{c}\right)-\frac{1}{3} \sin ^{3}\left(\theta_{c}\right)}{1-\cos ^{3}\left(\theta_{c}\right)} \\
& =\frac{9 \sqrt{3}}{7 \pi} \quad \text { with } \quad \theta_{c}=60^{\circ} .
\end{aligned}
$$

\section{Acknowledgments}

The authors would like to thank Professor Jean-Pierre Dalmont for helpful discussions during different stages of this work ; Alain Brunet and Eric Egon for their assistance in the experiments and their technical advices.

\section{References}

[1] F. C. Lindvall : A glow discharge anemometer. "I.E.E.E. 53 (1934) 1068-1073.

[2] L. Loeb : Basic processes of gaseous electronics. University of California Press, Berkeley, Los Angeles, 1961.

[3] M. Robinson : Movement of air in the electric wind of the corona discharge. Trans. Am. Inst. Elect. Engin. 80 (1961) 143-152.

[4] H. Yeh, H. Z. Cummins : Localized Fluid Flow Measurements with a He-Ne Laser Spectrometer. Appl. Phys. Lett. 4 (1964) 176-178.

[5] K. J. Nygaard : Electric Wind Gas Discharge Anemometer. Rev. Sci. Instrum. 36 (1965) 13201323.

[6] K. J. Nygaard : Anemometric characteristics of a Wire-to-plane electrical discharge. Rev. Sci. Instrum. 36 (1965) 1771-1774.

[7] Ph. M. Morse, K. U. Ingard : Theoretical acoustics. Princeton University Press, New Jersey, 1968.

[8] E. Nasser : Fundamentals of Gaseous ionisation and plasmas electronics. Wiley, New York, 1971.

[9] M. Abramowitz and I. A. Stegun : Handbook of mathematical functions. Dover publication, New York, 1972.

[10] W. L. Lama, C. F. Gallo : Systematic study of the electrical characteristics of the Trichel current pulses from negative needle-to-plane coronas. J. Appl. Phys. 45 (1974) 103-113.

[11] B. Desmet, M. Maton : Détermination expérimentale d'une impédance acoustique ne nécessitant que la mesure de pressions dans une ligne acoustique en série. C. R. Acad. Sci. Paris 280 (1975) 333-336. 
[12] K. Taylor : Absolute Measurement of Acoustic Particle Velocity. J. Acoust. Soc. Am. 59 (1977) 691-694.

[13] M. Goldman, A. Goldman and R. S. Sigmond : The corona discharge, its properties and specific uses. Pure \& Appi. Chem. 57 (1985) 1353-1362.

[14] M. Goldman, A. Goldman : Gaseous electronics, Ch.4 : Corona discharges. Academic Press, New York, 1978.

[15] J. Y. Chung, D. A. Blaser : Transfer Function Method of Measuring In-duct Acoustic Properties. I. Theory. J. Acoust. Soc. Am. 68 (1980) 907-913.

[16] J. Y. Chung, D. A. Blaser : Transfer Function Method of Measuring In-duct Acoustic Properties. II. Experiment. J. Acoust. Soc. Am. 68 (1980) 914-921.

[17] R. S. Sigmond : Simple approximate treatment of unipolar space-charge-dominated coronas The Warburg law and the saturation current. J. Appl. Phys. 53 (1982) 891-898.

[18] K. Janka : Ion deflection air flow meter with constant deflection. Rev. Sci. Instrum. 55 (1984) 976982.

[19] M. D. Yamanaka and H. Hirosawa and Y. Matsuzaka: Glow Discharge ionic anemometer. Rev. Sci. Instrum. 56 (1985) 617-622.

[20] P. A. Durbin, D. J. Mckinzie, E. J. Durbin : An anemometer for highly turbulent or recirculating flows. Experiments in Fluids 5 (1987) 184-188.

[21] R. J. Adrian : Statistical Properties of Particle Image Velocimetry Measurements in Turbulent Flow. Laser Anemometry in Fluid Mechanics, Lisbon (1988).

[22] A. D. Pierce : Acoustics - An Introduction to Its Physical Principles and Applications. Acoustical Society of America, New York, 1989.

[23] J. P. L'Huillier and C. Carol : A New Concept for the Mass Air Flow Rate Measurement The Electrostatic Compensation of the Ion Beam Deflection. Conférences Capteurs, France (1989) 170179 .

[24] J. P. Sharpe and C. A. Greated and C. Gray and D. M. Campbell : The Measurement of Acoustic Streaming Using Particle Image Velocimetry. Acustica 68 (1989) 168-172.

[25] R. S. Sigmond : Mass transfer in corona discharges. Rev. Int. Hautes Tempér. Réfract. 25 (1989) 201-206.

[26] Y. P. Raizer : Gas Discharge Physics. SpringerVerlag, Berlin, 1991.
[27] J. E. Jones : On the drift of gaseous ions. J. Electrostatics 27 (1992) 283-318.

[28] R. S. Sigmond and A. Goldman and M. Goldman : Ring vortex gas flow in negative point coronas. Proc. $10^{\text {th }}$ International Conference on Gas Discharges and their applications. Swansea, U.K. (1992) 330-333.

[29] R. S. Sigmond and I. H. Lågstad : Mass and species transport in corona discharges". Plasma Phys. Rep. 2 (1993) 221-229.

[30] H. H. Bruun : Hot Wire Anemometry - Principles and Signal Analysis. Oxford Science, Oxford, 1995.

[31] K. Asano, Y. Higashiyama, K. Yatsuzuka, K. Yamanaka: The behavior of emitted charge cloud from an axisymmetric ion-flow anemometer. J. Electrostatics 37 (1996) 39-52.

[32] F. J. M. Eerden, H. E. de Bree and H. Tijdeman : Experiments with a new acoustic particle velocity sensor in an impedance tube. Sensors and Actuators A 69 (1988) 126-133.

[33] J. C. Valière and Ph. Herzog and V. Valeau and G. Tournoi : Acoustic velocity measurements in the air by means of laser doppler velocimetry : dynamic and frequency range limitations and signal processing improvements. J. Sound. Vib. 229 (2000) 607-626.

[34] J.-P. Dalmont : Acoustic impedance measurement Part I : A review. J. Sound. Vib. 243 (2001) 427-439.

[35] E. O. Doebelin : Measurement Systems : Application and Design. Mc Graw-Hill, New-York, 2003.

[36] J. W. Van Honschoten, V. B. Svetovoy, T. S. J. Lammerink, G. J. M. Krijnen, M. C. Elwenspoek : Determination of the sensitivity behavior of an acoustic, thermal flow sensor by electronic characterization. Sensors and Actuators A 112 (2004) $1-9$.

[37] V. Joly : Etude de capteurs acoustiques à gaz ionisé. Ph-D Thesis Université du Maine, Le Mans, France, 2006.

[38] Ph. Béquin, V. Joly, Ph. Herzog: Modeling of a corona discharge microphone. J. Phys. D: Appl. Phys. 46 (2013) 175204.

[39] J-C Valière : Acoustic particle velocity measurements using lasers. Wiley, 2014.

[40] R. Bálek, M. Červenka, S. Pekárek: Acoustic field effects on a negative corona discharge. Plasma Sources Sci. Technol. 23 (2014) 035005. 
[41] Z. Zhou, J. D. Jones, A.M. Leung, W. Nie, X. Wang: A high-linearity DC planar ionic anemometer. Flow Measurement and Instrumentation $\mathbf{4 5}$ (2015) 118-125.

[42] Ph. Béquin, K. Castor and J. Scholten : Electric wind characterisation in negative point-to-plane corona discharges in air. Eur. Phys. J. AP. 22 (2003) 41-49.

[43] H. Becker, U. Kogelschatz, K.H. Schoenbach, R.J. Barker : Non-equilibrium air plasmas at atmospheric pressure. Institute of Physics Publishing, London, 2005. 